\title{
Pharmacovigilance: Perspectives in India
}

\author{
Novy Gupte ${ }^{1}$, Sapna Pradhan ${ }^{2}$
}

\begin{abstract}
Background: Adverse drug reactions are a significant burden on the health system across the world. Paradoxically, a considerable chunk of ADRs is missed or not reported on account of avoidable reasons, resulting in bottlenecks in carrying out the proper management. This problem is much more evident in India and other resource-limited communities than in the prosperous countries of the West.

Aim and objective: To provide a state-of-the-art update on pharmacovigilance with special reference to perspectives in India and make appropriate recommendations for improving ADR reporting.

Design: The contents are based on a review of English medical literature augmented with the author's first-hand experience in the field over the past more than a decade.

Results: Well-organized pharmacovigilance started in India with the launching of the Pharmacovigilance Programme of India in 2010 . Despite it making a perceptible improvement in ADR reporting, high underreporting of ADRs still prevails. The yet greater thrust on inculcating awareness comprising knowledge attitudes and practices among the healthcare professionals is likely to lead to further improvement in ADR reporting, resulting in augmentation of the safe use of drugs.
\end{abstract}

Conclusion: Pharmacovigilance, a mandatory drug safety activity, needs to be propagated with yet greater vigor among healthcare professionals in India.

Keywords: Adverse drug events, knowledge, attitudes, and practices, Adverse drug reactions, Pharmacogenomics, Pharmacovigilance, Pharmacovigilance program of India, Stakeholders.

Journal of Medical Academics (2020): 10.5005/jp-journals-10070-0062

\section{INTRODUCTION}

Adverse drug reactions (ADRs) are a significant burden on the health system across the world. ${ }^{1-4}$ It is often labeled as one of the top causes of morbidity and mortality in health facilities. Paradoxically, a considerable chunk of ADRs is missed or not reported on account of avoidable reasons, resulting in bottlenecks in carrying out the proper treatment. This problem is much more evident in the resource-limited communities than in the prosperous countries of the West.

"Pharmacovigilance", through its proactive activities, has turned out to be a "paradigm" for ensuring the safe and appropriate use of drugs, resulting in drug safety.

This research-cum review communication provides an update on perspectives in pharmacovigilance with special reference to India punctuated by our own experience in the field over more than a decade.

\section{Definition and Role}

Pharmacovigilance (PVG) deals with the complete study of ADRs. "Pharmakon" denotes "Drug". "Vigilance" means "keeping watch or alert".

The World Health Organization (WHO) defines pharmacovigilance as "the science and activities relating to the detection, assessment, understanding, and prevention of adverse effects or any other possible drug-related problems". ${ }^{5}$

Landmarks in the development of pharmacovigilance are listed in Box 1.

The success of pharmacovigilance banks on positive interactions among stakeholders, decision-making authorities, and the medical profession responsible for patient safety and welfare.

Precisely, the role of pharmacovigilance is three-fold (Box 2).
1,2Department of Pharmacology, Army College of Medical Sciences, New Delhi, India

Corresponding Author: Novy Gupte, Department of Pharmacology, Army College of Medical Sciences, New Delhi, India, Phone: +91 8178971105, e-mail: drnovyguptemd@gmail.com

How to cite this article: Gupte N, Pradhan S. Pharmacovigilance: Perspectives in India. J Med Acad 2020;3(2):59-62.

Source of support: Nil

Conflict of interest: None

Pharmacovigilance is important to ensure the safe use of medicines. Teaching pharmacovigilance to medical students makes them aware of their responsibility to report ADRs. The teaching

Box 1: Landmarks in the development of pharmacovigilance

1937 Sulfonamide disaster. This comprised death of over 100 patients who consumed a sulfonamide (sulphanilamide) dissolved in diethylene glycol from acute renal failure. Under the existing drug regulations, premarketing toxicity testing was not mandatory.

1938 Food and Drug Organization (FDA) made the preclinical toxicity and pre-marketing clinical studies mandatory.

1950s Chloramphenicol was found causing aplastic anemia. 1960 FDA launched a hospital-based drug monitoring program. 1961 Thalidomide tragedy: The drug damaged around 10,000 embryos during pregnancy. About $40 \%$ died around the time of birth. The survivors suffered from various birth malformations, including phocomelia.

1963 Rapid action on ADRs recommended by Sixth World Health Assembly. 1968 International Drug Monitoring Program by the WHO established. 
Box 2: Three-fold role of pharmacovigilance

- Identify, quantify and document drug-related

- Contribute to reduce the risk of drug-related

- Increase knowledge and understanding of factors and mechanisms which are responsible

should be mainly problem-based, activity-based, and carried out in small groups. Medical officers and interns should be made aware of reporting systems in their hospitals. Doctors, from other specialties, have an important role in reporting ADRs and sensitizing medical students to the importance of pharmacovigilance. Pharmacologists have the primary responsibility of running a pharmacovigilance program. Postgraduate pharmacology students should be actively involved in the running of a pharmacovigilance center.

\section{Pharmacovigilance AND Pharmacogenomics ${ }^{6}$}

Advances in genetics have opened new avenues in pharmacotherapy in the form of what is now termed "pharmacogenomics". 7 It is the study of pharmacologically relevant genes, their variation, the modus operandi of the variations that interact to produce phenotypes, and the modus operandi of their phenotypes on the drug response. The study of pharmacogenomics, hopefully, may increase the predictability of drug response in individual patients, thereby reducing ADRs. It may also help in improving effectiveness as response rates with most drugs are between 25 and $60 \%$.

The ultimate aim of pharmacogenomics is to go largely from the "one-size fits all" paradigm of drug prescription to tailored medication regimens based on a patient's genetic characteristics. In other words, pharmacogenomics could help "individualize" drug therapy and become one of the pillars of "personalized medicine".

Thus, ADRs that were previously considered to be beyond prevention may now be rendered preventable through modification of drug selection and/or dosage in patients based on their genotype.

\section{Pharmacovigilance in India ${ }^{8,9}$}

\section{India as a Drug Hub}

India is fast heading for the distinction of being the "Global pharmacy of Generic Drugs". Further, India is also fast emerging as a "hub" of global clinical trials and a destination for new drug discovery and development. Additionally, an increasing number of new drugs are being introduced into the country, including new chemical entities (NCEs), high tech pharma products, vaccines, etc. Moreover, new dosage forms, new routes of drug administrations, and new therapeutic claims of existing drugs are on the way. This is reflected in the fact that the total number of applications received and processed have hiked now by several folds from around 10,000 in the Year 2005 at CDSCO headquarters, New Delhi. This includes an increase in New Drug Applications, Global Clinical Trials, Market Authorization of Vaccine and Biotech products.

India is the world's second most populated country with over 1.35 billion potential drug consumers and a value of US $\$ 1.4$ billion worth of clinical trials.

\section{India's Need for Drug Monitoring}

By and large, all medicines have ADRs. Some of these ADRs are known. However, some are still unknown even though the
Box 3: Objectives of the PvPI

Short-term objectives: To foster a culture of notification

Medium-term objectives: To engage several healthcare professionals and nongovernmental agencies (NGOs) in the drug monitoring and information dissemination processes.

Long-term objectives: To achieve such operational efficiencies that would eventually achieve safety in use of drugs.

concerned medicines may have been in clinical use for several years. Hence, it is important to monitor both the known and hitherto unknown ADRs of medicines to determine any new information available in relation to their safety profile.

Understandably, such a rapid induction of NCEs and high tech pharma products in the market and their use in the patients throw up the challenges of monitoring the ADRs over a large population base through a standardized and robust system and drug safety monitoring program. Systematically collecting this information and analyzing the data to reach a meaningful conclusion on the continued use of these medicines is the rationale to institute this program for India. Since there are considerable social and economic consequences of ADRs there is a need to engage health-care professionals, in a well-structured program to build synergies for monitoring ADRs.

Adverse drug reaction reporting has been far from adequate in India. Doctors are reluctant to report them. The Ministry of Health in India sites a "culture of reluctance in reporting" as the problem. That may well be true. However, an unwritten factor is an apprehension and fear on the doctors' parts that they could land into medical-legal troubles.

\section{Pharmacovigilance Programme of India (PvPI)}

It was way back in 1986 that pharmacovigilance in India was initiated with a formal ADR monitoring system, under the supervision of the Drug Controller of India. Subsequently, in 1998, India joined the WHO Programme for International Drug Monitoring. Somehow it could not make a substantial grade. In 2004, National Pharmacovigilance Programme was initiated with the All India Institute of Medical Sciences (AIIMS) as the coordinating center but unfortunately, it could not make a big difference.

Formally, the PvPI was launched on July 14, 2010. Initially, the AIIMS, New Delhi was the National Coordination Centre (NCC) for monitoring ADRs in the country for safeguarding drug use. In 2011, PvPI adopted Indian Pharmacopeial Commission (IPC), Ghaziabad as the NCC. Since then, there has been rapid progress in reporting of ADRs by the healthcare professionals.

The launching of the Pharmacovigilance Programme in India is aimed at collecting, and analyzing data to arrive at an inference to recommend regulatory interventions, besides communicating risks to health care professionals and the public at large.

The objectives of the PvPI are listed in Box 3.

Meanwhile, over the past decade, several scores of teaching hospitals and corporate hospitals have been approved as ADRs Monitoring Centres (AMCs). The AMCs report to IPC. Indian Pharmacopeial Commission finally connects with International Networking Reporting WHO-Uppsala Monitoring Centre (UMC). The entire reporting is done through the software named VigiFlow.

Undoubtedly, ADRs reporting through PvPI endeavors improved with the measures such as education, training, and provision of technical assistance. 


\section{Current Challenges}

The efforts of the PvPI have cut down the level of reluctance in reporting ADRs on the part of the health professionals to some extent. However, reluctance in ADR reporting continues to be a real bottleneck though to a less extent with time.

This "reluctance" is significantly related to a lack of awareness among the health professionals who are primarily involved in reporting, monitoring, and prevention of ADRs. Naturally, drug safety issues remain to be more vigorously pursued.

Our own study carried out in four medical colleges in Lucknow shows that a large proportion of the doctors have inadequate knowledge about pharmacovigilance. ${ }^{10}$ To a marginally larger extent, attitudes too are faulty. As far as the practices are concerned, even doctors with reasonable knowledge and attitudes are lacking in sound practices. Several other studies have more or less similar findings with some variations. ${ }^{11-24}$

We strongly feel that the PvPI needs to expand its scope of activities to widen its reach to much larger healthcare professionalism with greater vigor and to strengthen measures for capacity building.

\section{ADR Reporting: Practical Considerations}

All health professionals, especially doctors and nurses, must be sensitive to the detection of ADRs and their reporting appropriately.

The PvPI encourages reporting of all suspected drug-related adverse reactions, including adverse events related to vaccines and reactions suspected to have been caused by herbal, traditional, or alternative remedies.

Even the reporting of apparently minor and insignificant or common adverse reactions is important.

The information provided in the submitted form is analyzed for "causality" at AMCs by using the WHO-UMC scale. Thereafter, the analyzed forms are forwarded to the NCC through the ADR database in the final step, the data are analyzed and forwarded to the Global Pharmacovigilance Database managed by WHO Uppsala Monitoring Centre in Sweden.

\section{Summary, Conclusion, And Recommendations}

Well-organized pharmacovigilance, though in operation in Western countries since long, started in India with the launching of the PvPI way back in 2010. The activities of PvPI have made perceptible improvement in ADR reporting. However, high underreporting of ADR still prevails. The yet greater thrust on inculcating awareness among the health professionals is likely to boost the knowledge, attitudes, and practices of the healthcare professionals and lead to further improvement in ADR reporting, resulting in augmentation of the safe use of drugs.

- Ensuring the creation of a separate unit of pharmacovigilance under the department of pharmacology.

- Thus, established pharmacovigilance should develop a rapport with the clinical doctors and nurses in particular who actually observe drug reactions.

- The frequent holding of educational and sensitization seminars, symposia, and workshops on pharmacovigilance with special emphasis on ADR reporting.

- Provision of the pharmacovigilance teaching in the undergraduate curriculum.
- Involvement of other stakeholders, including NGOs in pharmacovigilance activities.

\section{ACKnOWLedgments}

The author wishes to record her indebtedness to $\mathrm{Dr}$ (Maj-Gen) SM Sharma, Dean and Dr (Lt Col) AK Gupta, Professor and Head of the Department of Pharmacology, Army College of Medical Sciences, New Delhi, for permission to publish this article.

\section{References}

1. Gupte N, Gupta S, Kumar H, et al. Causality assessment in cancer chemotherapy-induced adverse drug reactions in a tertiary-care centre in North India. ECPharmacol Toxicol 2010;7:74-85.

2. Tandon VR. Undereporting of adverse drug reactions: a challenge for pharmacovigilance in India. Indian J Pharmacol 2015;47(1):65-71. DOI: 10.4103/0253-7613.150344.

3. Abadie D, Chebane L, Bert M, et al. Online reporting of adverse drug reactions: a study from a French regional pharmacovigilance center. Therapie 2014;69(5):395-400. DOI: 10.2515/therapie/2014035.

4. Rubina Mulchandania R, Kakkarb AK. Reporting of adverse drug reactions in India: a review of the current scenario, obstacles and possible solutions. Int J Risk Safe Med 2019;30(1):33-34. DOI: 10.3233/ JRS-180025.

5. World Health Organisation, Pharmacovigilance. Available a: https:// www.who.int/medicines/areas/quality_safety/safety_efficacy/ pharmvigi/en/ Accessed on: 20 December 2020.

6. Sahu S, Rishishwar P, Rathod C. Pharmacovigilence practice for safety of medication system in India. J Phytopharmaco 2018;7:216-221.

7. National Humam Genome Research Institute, Pharmacogenomics FAQ. Available at: https://www.genome.gov/FAQ/Pharmacogenomics Accessed on 22 December 2020.

8. Suke SG, Kosta SG, Negi H. Role of pharmacovigilance in India. Online J Publ Health 2015;7:e23.

9. Kalaiselvan V, Thota P, Singh GN. Pharmacovigilance Program of India: recent developments and future perspectives. Indian J Pharmacol 2016;48(6):624-628. DOI: 10.4103/0253-7613.194855.

10. Gupte N, Garg HK. Pharmacovigilance-related "knowledge, attitudes and practices among institutional doctors: questionnaire-based study. JEC Pharmacology Toxicol (London), In press.

11. Ganesan S, Vikneswaran G, Reddy KC, et al. A survey on knowledge, attitude and practice of pharmacovigilance towards adverse drug reactions reporting among doctors and nurses in a tertiary care hospital in South India. J Young Pharm 2016;8(4):471-476. DOI: 10.5530/jyp.2016.4.25.

12. Desai CK, lyer G, Panchal J, et al. An evaluation of knowledge, attitude, and practice of adverse drug reaction reporting among prescribers at a tertiary care hospital. Perspectives in Clin Res 2011;2(4):129-136. DOI: 10.4103/2229-3485.86883.

13. Gupta SK, Nayak RP, Shivaranjani R, et al. A questionnaire study on the knowledge, attitude, and the practice of pharmacovigilance among the healthcare professionals in a teaching hospital in South India. Perspect Clin Res 2015;6(1):45-52. DOI: 10.4103/2229-3485.148816.

14. Khan SA, Goyal C, Chandel N, et al. Knowledge, attitudes, and practice of doctors to adverse drug reaction reporting in a teaching hospital in India: an observational study. J Nat Sci Biol Med 2013;4(1):191-196. DOI: 10.4103/0976-9668.107289.

15. Health Professionals' Knowledge, attitudes and practices about Pharmacovigilance in India: A systematicreview and meta-analysis. Available at:: http://journals.plos.org/plosone/ Accessed on: 25 December 2020.

16. Srinivasan V, Sheela D, Mridula D. Knowledge, attitude, and practice of pharmacovigilance among the healthcare professionals in a tertiary care hospital - A questionnaire study. Biomed Pharmacol J 2017. 10. Available at http://biomedpharmajournal.org/?p=16563. Accessed on: 25 December 2020. 
17. Kharkar M, Bowalekar S. Knowledge, attitude and perception/ practices (KAP) of medical practitioners in India towards adverse drug reaction (ADR) reporting. Perspect Clin Res 2012;3(3):90-94. DOI: 10.4103/2229-3485.100651.

18. Gupta $P$, Udupa A. Adverse drug reaction reporting and pharmacovigilance: knowledge, attitudes and perceptions among the resident doctors. J Pharm Sci Res 2011;3:1064-1069.

19. Chopra D, Wardhan N, Rehan HS. Knowledge, attitude and practices associated with adverse drug reaction reporting amongst doctors in a teaching hospital. Int J Risk Saf Med 2011;23: 227-232.

20. Ramesh M, Parthasarathi G. Adverse drug reaction reporting: attitudes and perceptions of medical practitioners. Asian J Pharm Clin Res 2009;2:10-14.
21. Chandrakapure AR, Giri SP, Khan IN, et al. Pharmacovigilance: a study to evaluate knowledge, attitude, and practices of and impact of educational intervention among doctors in teaching hospital, in rural area of Jalna, India. Int J Basic Clin Pharmacol 2015;4:427-431.

22. Arbind KC, Nivedhitha $S$, Manicvasagam $S$, et al. Awareness and perception experiences on adverse drug reaction among doctors, nurses and pharmacists of a tertiary care rural teaching hospital. Indian J Drugs Dis 2013;2:248-258.

23. Kulkarni MD, Baig MS, Chandaliya KC, et al. Knowledge, attitude and practice of pharmacovigilance among prescribers of Government Medical College and Hospital, Aurangabad (Maharashtra). Int J Pharm Ther 2013;3:10-18.

24. Hema NG, Bhuvana KB, Sangeetha. The extent of awareness among the final year students, interns and postgraduates in a government teaching hospital. J Clin Diagn Res 2012;6(Suppl. 7):1248-1253. 